\title{
10/11 images
}

Catherine PARAYRE, Université Brock

poteaux télégraphiques

jetés contre un ciel gris

- cure-dents dans un cendrier de métal

(Alexandre Amprimoz, 10/11 «c/10»)

Alexandre Amprimoz commence ses Changements de tons (1981) par une explication de la «parole» : celle-ci est prononcée «souvent au sein du cantique / le bourdonnement de la cascade / et l'ignorance du vent» («Mots», 11). Au lieu d'exprimer ce qu'elle est, cette description situe la parole dans un espace à la fois intérieur et paysager. De fait, les images du dedans et des alentours abondent dans l'œuvre du poète et forment un trésor d'étonnantes évocations. Dans 10/11 (1979), les images du dedans sont enfouies dans des cendriers, des océans, des masques, des palais de marbre, entre autres exemples, alors que l'environnement convoque les plaines neigeuses, de belles terres, des iles, des jardins de fleurs, «des soleils en rayons » $(11 / \mathrm{p})$, une ville avec sa gare et son université, etc.

\section{Symbolismes dessus-dessous}

De toute évidence, cette poésie travaille et transforme des motifs et images chers à Arthur Rimbaud, Charles Baudelaire et autres symbolistes. En voici quelques exemples qui semblent bel et bien faire écho aux illustres prédécesseurs dont Amprimoz aimait tant la poésie. Commençons avec la femme-paysage de Baudelaire dans «Parfum exotique»: «Quand, les deux yeux fermés, en un chaud soir d'automne / Je respire l'odeur de ton sein chaleureux, / Je vois se dérouler des rivages heureux / Qu'éblouissent les feux d'un soleil monotone »(38). Chez Amprimoz, la promenade sur les plages exotiques se transforme en balade dans un climat plus tempéré : «je veux parcourir les chemins de ton corps / (fourmi en pèlerinage voulant faire le plein de tes saintes saveurs) / je pars avec le peuple humide / des coquelicots qui vibrent / sur tes lèvres »(a/11). Tant qu'on en est aux fourmis, aussi bien relire «Les sept vieillards »de Baudelaire qui s'effraie de la vieillesse : «Fourmillante cité, cité pleine de rêves, / Où le spectre en plein jour raccroche le passant ! / [...] / je comptai sept fois, de minute en minute / ce sinistre vieillard qui se multipliait ! / [... Mais je tournai le dos au cortège infernal. » (104-105). Chez 
Amprimoz, le cortège funèbre devient la procession d'un baptême et, pour ne plus être confronté au spectacle désolant des ravages du temps, Amprimoz propose tout bonnement de faire disparaitre les nouveaux nés. La cité fourmillante est à présent une fourmilière : «c'est la fourmi qui couche avec le lion / on alla au baptême du fourmilion / c'est fou mais le pauvre enfant fut mangé / par le curé qui était bien entendu un fourmilier » (10/r).

Dès son adresse «Au lecteur », Baudelaire inventoriait «les chacals, les panthères, les lices, / Les singes, les scorpions, les vautours, les serpents, / Les monstres glapissants, hurlants, grognants, rampants / Dans la ménagerie infâme de nos vices » (18). Amprimoz a lui aussi sa ménagerie, mais même si ses animaux font parfois preuve de cruauté, celle-ci s'accompagne d'un sourire absent de la poésie symboliste : «sur ma planche à dessin il y a / des colombes jalouses des tourterelles / empoisonneuses et des pélicans / égoïstes / tandis que les scorpions / s'en vont deux par deux / comme des amoureux » (10/h). Le poète est lui-même un pensionnaire de ce petit zoo : «le calmar cet écrivain / nous parle d'un paradis / où les hiboux chevauchent / des hippocampes » $(\mathrm{m} / 10)$.

Tout aussi bien, les instants de mysticisme, ainsi un fameux vers de Baudelaire dans «Harmonie du soir »- «Voici venir les temps où vibrant sur sa tige / Chaque fleur s'évapore ainsi qu'un encensoir » (61) - n'évoquent plus dans la mouture amprimozienne le temple de la nature, mais le commerce - de toutes sortes - hors de ses piliers : «voici venir le marchand / chaque bouteille est un calice / qui fait fleurir ma tige » $(u / 11)$.

Très frappantes, de nombreuses images baudelairiennes émotionnellement chargées naissent de la description des meubles qui, sous la plume du poète, recèlent un monde en miniature, un cabinet de curiosités et tout le drame d'une vie. Il est ainsi « Des meubles luisants, / Polis par les ans » (68) dans «L'invitation au voyage » ou, dans «Une martyre », « des flacons, des étoffes lamées / Et des meubles voluptueux » (130). Rimbaud inverse cette image du tropplein sans en enlever la portée affective, dans «Les étrennes des orphelins » où l'armoire a perdu ses «mystères dormant entre ses flancs de bois » après la mort des parents; elle est maintenant «béante», «bien vide» (3-6). L'armoire figure également dans le mobilier poétique d'Amprimoz, mais sans sentimentalité : «l'armoire a le ventre plein / sourire rose / d'un évêque blanc / l'armoire a le ventre vide / grimace verte / d'un pape noir » (o/10).

Quant au spleen qui traverse l'œuvre de Baudelaire, écoutons comment il se traduit dans 10/11. C'est celui des «arbres aux branches maigres » qui ressemblent aux «cheveux dans la 
sale baignoire du ciel » $(\mathrm{c} / 10)$. Baudelaire se montre bien plus sobre dans ses descriptions des jours de pluie. Point de baignoire dégoutante où trainent de longs cheveux : «Quand le ciel bas et lourd pèse comme un couvercle»; «Quand la pluie étal[e] ses immenses traînées », «de longs corbillards, sans tambours ni musique, / Défilent lentement dans mon âme » (90). Dans le petit poème d'Amprimoz, les corbillards ont quitté la ville et laissé place à un moyen de transport moins lugubre: «je suis seul / comme une grande / gare/ les trains / sont des cigares / dans la bouche bleue / de l'horizon » (q/11). Le ciel bleu au-dessus de la gare, la gare elle-même où on a le loisir de fumer, les trains qui évoquent voyages et aventures et pas uniquement de tristes occasions, sont moins sinistres que les identifications du soi chez Baudelaire, à savoir «Je suis comme le roi d'un pays pluvieux» (89). «Je suis un cimetière abhorré de la lune »; «Je suis un vieux boudoir plein de roses fanées » (88).

Une pareille transformation s'applique aussi à des citations de Rimbaud. Dans une admirable illumination, les gouffres de l'océan nourrissent l'imagination du poète comme une sucrerie aux saveurs inaccoutumées: "J'ai rêvé la nuit verte aux neiges éblouies / Baiser montant aux yeux des mers avec lenteurs / La circulation des sèves inouïes, / Et l'éveil jaune et bleu des phosphores chanteurs » (67). Chez Amprimoz, les abysses des océans ressemblent plutôt à des poubelles : «tu regardes ces phares - / cigarettes dans le cendrier de la mer » (g/10). Selon Rimbaud, et sur un ton plus harmonieux, le poète est «Libre, fumant, monté de brumes violettes, / Moi qui trouais le ciel rougeoyant comme un mur / Qui porte, confiture exquise aux bons poètes, / Des lichens de soleil et des morves d'azur » (68). Dans un changement de décor, la confiture rimbaldienne de toutes les excellences réapparait chez Amprimoz, cette fois du fond d'un lit confortable dans une chambre qui n'est peut-être pas très propre : «la confiture de tes paroles / s'étale sur les murs / la marmelade de tes pensées / coule du plafond / ton esprit fond au cœur d'un lit / de pavots blancs » (10/p).

Un second exemple renvoie au délicat «Clair de lune» de Paul Verlaine : «Votre âme est un paysage choisi / Que vont charmant masques et bergamasques / [...] / Au calme clair de lune triste et beau, / Qui fait rêver les oiseaux dans les arbres / Et sangloter d'extase les jets d'eau, / Les grands jets d'eau sveltes parmi les marbres » (107). En écho, Amprimoz renchérit : « frêle / le souffle des fontaines / murmure parmi les arômes du soir / - échos douloureux de rires cristallins » (10/z). Toutefois, notre poète n'hésite pas à laisser ce paysage raffiné se figer; la scène se solidifie et devient cauchemardesque : les « Visages [sont] ensevelis / dans l'épaisseur 
des masques / où se déforment / les paroles / [...] / dans une boue de confetti » (10/f). Ce processus de pétrification est loin d'être élégant : «vos visages / épongent / la lumière / faisant l'hiver / dans les palais de marbre » (i/10).

Amprimoz ne rechigne pas à l'occasion à faire un clin d'œil aux auteurs du vingtième siècle, par exemple dans le poème a/10. Tout comme les vers "pommes aux rides maquillées / poires boutonneuses» pourraient s'intégrer à une rengaine amusamment injurieuse qui inverserait l'éloge de la femme dans «Union libre » (1931) d'André Breton, les deux vers suivants semblent également procéder à une permutation. En effet, «sur les pépins de vos fatigues / j'abîme mes dents » réinscrivent en creux la litanie des «J'écris ton nom » dans «Liberté » (1942) de Paul Eluard, en particulier en référence au vers «Sur la santé revenue » (np.).

\section{L'humour est triste}

Comme on sait qu'Amprimoz était un lecteur passionné des poètes symbolistes (et autres) ainsi qu'un esprit joueur et enjoué, la tentation est effectivement grande pour nous ses lecteurs de nous prêter à ce petit jeu d'identifications et d'imaginer que notre poète aurait un sourire amusé s'il nous y surprenait. L'humour qui habille ses poèmes, est une invitation aux lecteurs à jouer ce jeu et à voir les petits poèmes de 10/11 comme autant d'échos déformants de célèbres vers poétiques d'un autre siècle, ceux-ci plus solennels, réservoirs de sentiments profonds et des tracas de poètes renommés pour avoir cherché la beauté, la laideur et le nouveau en toute chose.

Point ne s'en faut néanmoins d'attribuer par contraste aux poèmes d'Amprimoz une légèreté facile qui se résumerait à une simple moquerie ou au travestisme des maitres. Comme l'explique Dominique Noguez dans «L'humour ou la dernière des tristesses », l'humour est « une forme équivoque » (140) et l'humoriste, « cet ennemi du sérieux[,] est très sérieux » (148). De nombreuses années plus tard, Christian Morin reprend ce propos dans « Pour une définition sémiotique du discours humoristique » et précise : «L'action humoristique prend [...] sa source dans le malheur et s'y frotte continuellement jusqu'à sa fin »(93). L'humour consisterait à «jouer par l'invention et l'innovation pour ne pas désespérer ou plus exactement jouer pour dépasser le désespoir » (92). Voici ce qu'écrivait Noguez en 1969 : «Ce qui pèse à l'humoriste, c'est ce qui est déjà-là. C'est tout ce qui, sans lui, avant lui, s'est édifié, et désormais l'écrase. C'est la lourdeur monolithique du sérieux, l'architecture compacte des habitudes et des 
contraintes, les tabous, les rituels, les pudeurs - tout ce qui, de nature ou de coutume, limite et retient » (140). Il dit de l'humoriste : «s'il futilise le grave et aggrave le futile, c'est qu'il est au plus haut point sensible, lui, à la gravité de ce qui est grave, à la futilité du futile. Intervertir les valeurs en signalant qu'on les intervertit, c'est encore le moyen le plus sûr d'indiquer leur vraie place. C'est le contraire de l'indifférence » (141).

Les éléments les plus hétéroclites qui habitent les paysages de 10/11 et meublent les intérieurs de ses maisons, produisent une impression amusante, mais aussi infiniment triste comme si ces objets et leur environnement avaient à la fois perdu leur fonction poétique - c'està-dire leur fonction d'enchantement - et leur raison d'être dans un monde devenu dérisoire. Par ailleurs, le poète semblerait avoir perdu son rôle de voyant génial et de frère doué de sensations extraordinaires dans les forêts de symboles qui nous entourent. Le voyant, le poète aurait perdu l'acuité de ses sens, ce qui l'amènerait à placer les objets dans des endroits inopportuns. De fait, le renversement de tout semblant d'ordre est un principe essentiel de l'humour (Morin 91), qui est un jeu consistant à « désassemble[r] les pièces connexes du rouage, et [...] bientôt démolit la machine » (Noguez 142). Les poèmes que contiennent 10/11 investissent certains motifs du symbolisme et les rendent caduques tout en leur assignant une contemporanéité consciente de ce que Noguez nomme les «grains de sable » qui défont les mécanismes bien huilés de l'écriture (142).

\section{Affinités visuelles}

De toute évidence, Amprimoz se délecte à créer des affinités et autres cousinages littéraires, mais ses poèmes gagnent aussi à être lus au-delà des problématiques de la littérature et de ses mouvements, en-dehors donc des horizons intertextuels. Si on peut, comme Roland Barthes le recommande, «étoiler» (563) les textes afin d'y observer «la migration des sens, l'affleurement des codes, le passage des citations » (564), « sans jamais aller jusqu'à construire ou reconstruire - la culture qu'ils articulent» (568), on sera sans doute tenté, dans ce même esprit, d'élargir le champ d'investigation, notamment de l'ouvrir aux perspectives de l'expression visuelle et, de la sorte, à des échos «plus volatiles et plus complexes » (Bryson 15) que ceux qui se forment dans une seule discipline. Cet exercice se révèle gratifiant dans une poésie aussi incisive que celle d'Amprimoz. 
Les petits textes de 10/11 sont, n'en doutons pas, le produit d'une esthétique visuelle toute contemporaine caractérisée par une indéniable impassibilité dans la pratique artistique d'un humour pince-sans-rire qui connait depuis les années soixante une grande popularité. La critique Charlotte Cotton la définit comme un ensemble de techniques sophistiquées, voire « encyclopédiques » (82), en tout cas nourries d'une connaissance approfondie de traditions plus anciennes dans les arts visuels. Cette esthétique visuelle révèle, d'une part, une grande maitrise de l'expression et du potentiel «élégiaque» (93) des images, et, d'autre part, traduit une intention de détachement et une volonté analytique, plutôt qu'un souhait de manifester des sentiments ou impressions sollicitant une réponse empathique. Très prisée, cette esthétique contemporaine permet à ses adeptes de créer des œuvres fortes en effets visuels qui résistent aux épanchements et convient le public à savourer leur formidable ingéniosité (81).

Les exemples abondent et certains, particulièrement représentatifs de l'esprit des années pendant lesquelles Amprimoz écrit, pourraient en toute légitimité illustrer ses vers. Je me servirai des analyses de Derek Knight pour exemplifier cette approche et commencerai, comme lui ( Post-Modernism», 11), avec la comparaison établie par Fredric Jameson entre Les vieux souliers (1885-1887) de Vincent van Gogh et Diamond Dust Shoes (1980-1981) d'Andy Warhol. Reprenant cet exemple, le critique Paul Wood déclare que l'œuvre de Warhol montre « une nouvelle catégorie de plat ou absence de profondeur, une nouvelle catégorie de superficialité dans son sens le plus littéral » (19; ma traduction). Pour nous tenir à une inspiration plus proche du symbolisme qui motivait Amprimoz, considérons le travail de deux artistes canadiens qui ont adapté Un bar aux Folies Bergère (1882) d'Edouard Manet, chronologiquement Michael Snow avec Authorization (1969), photographie dans laquelle l'artiste pose avec son appareil polaroid face à un miroir, debout et fixant l'appareil droit devant lui, comme la serveuse du célèbre tableau, son dos reflété par le miroir mural, et Jeff Wall avec Picture for Women (1979) et ses deux personnages, l'un une jeune femme dans la même posture que la serveuse des Folies Bergère, l'autre le photographe installant son appareil et préparant la scène. Incidemment, Knight note des premières œuvres de Wall qu'elles ont été caractérisées d'œuvres baudelairiennes dans lesquelles il fait apparaitre par l'un ou l'autre symbole la désolation de l'existence («Trends», 7). Dans les deux exemples cités, Snow et Wall mettent à nu l'appareillage technique nécessité pour créer une œuvre d'art ; la scène suggérant le tableau de Manet est proposée comme prétexte au travail technique (11-12). En particulier, l'œuvre 
photographique de Wall est « basée sur des techniques mnémoniques appliquées à l'histoire de la peinture, et met ainsi en relief le passage du modernisme au postmodernisme » (7; ma traduction). En poésie, Amprimoz procède de manière similaire; les échos symbolistes lui permettent de créer de petits textes rythmés comme des comptines contemporaines.

C'est le cas, entre autres, de ses personnages animaliers dont le traitement plaisant n'est guère différent de ce que divers artistes produisent à la même époque, par exemple Jeff Koons dans la représentation de Michael Jackson tenant dans ses bras son chimpanzé Bubbles (1988), œuvre kitsch qui évoque également l'art statuaire grec et roman, normatif jusqu'à la fin du dixhuitième siècle («Post-Modernism »; 18). Les installations de Snow, Flight Stop (1979), avec ses fières bernaches du Canada qui décorent les arches transparentes d'un centre commercial de Toronto, et, quelques années plus tard, de General Idea avec Snowbird (1985), pastiche d'oiseaux blancs faits de boites de plastique, en sont des exemples supplémentaires. Ils illustrent la déclaration de Jameson, selon laquelle le modernisme est ce qu'il reste une fois la nature disparue (ix), mais aussi combien une œuvre peut rapidement être suivie par sa caricature («Trends »; 20-21). Qu'on ne s'y méprenne ; comme dans l'humour d'Amprimoz, ce recyclage visuel reste souvent empreint de sérieux, par exemple dans Fin de siècle (1994) de General Idea avec ses phoques innocents dont la vulnérabilité est renforcée, d'une part, par la référence à l'isolation ressortant du célèbre tableau de Casper David Dietrich, La mer de glace (1824), et, d'autre part, du fait que General Idea a réalisé cette imitation en polystyrène pour nous alarmer des dangers de la pollution (22).

Notre poète aime également les comparaisons osées. A l'idée du calice «qui fait fleurir [1]a tige » du poète amprimozien, pourquoi ne pas penser à son contemporain Robert Mapplethorpe dans Calla Lily (1984), tirage argentique montrant la longue tige d'un lys et sa corolle, et, dans une nouvelle mouture humoristique, à Untitled XIII (Ejaculate in Trajectory), (1989) d'Andres Serrano (voir Knight, «Feminism»), des œuvres qui ont suscité de violents débats? A propos du corps humain, la vision qu'en a Amprimoz, semble proche de celle de Joyce Wieland dont l'autoportrait Artist on Fire (1983) représente expressivement un enthousiasme exubérant lors d'une rêverie émancipée (2) ou, lorsqu'il s'agit indirectement de souffrance ( «Trends »; 15), de celle de l'artiste Betty Goodwin dans Red Sea (1984).

Finissons avec les neiges éblouies dont Rimbaud remarque les teintes jaunes, bleues et mauves, les lichens brillant sur l'eau et les trainées d'azur, et sur ce qu'en retient Amprimoz, à 
savoir des paroles en confiture qui s'étalent et coulent, et des fleurs blanches sur tige n'oublions pas les paysages du nord qu'Amprimoz aimait tant. Notre poète a recours au même processus de recyclage qu'adopte Snow vis-à-vis de l'iconique Jack Pine (1916-1917) de Tom Thomson. Snow a pris cette série de photos lors de l'exposition du Groupe des sept en 1977 alors qu'il traversait les salles d'un pas rapide, ce qui a pour effet de mettre en cause l'importance généralement incontestée du Groupe des sept dans l'art canadien («Trends»; 5). En 1990, son Pine on the Corner, exemplaire dressé au-dessus d'un quartier quelconque en contraste avec la splendeur de la nature du Groupe des sept, reproduit une semblable démarche.

\section{Conclusion entre légèreté et lucidité}

Cette mise en perspective de textes d'Amprimoz par rapport à ce qu'on pourrait appeler l'air du temps au moment de leur écriture, met en relief certains effets caractéristiques de son art poétique. Tout d'abord, sa réappropriation d'images symbolistes s'articule sur la qualité élégiaque de la poésie du dix-neuvième siècle sans pour autant creuser cet élégiaque et en remplir ses textes. Bien au contraire, toute impression de mélancolie s'estompe dans la légèreté des vers, qui donnent ainsi une illusion de détachement envers le sujet traité. Leurs images du dedans ne recèlent aucun trésor de beauté, comme chez Baudelaire qui la cherche au fond du mal, et celles du dehors ne formulent aucune recherche du nouveau, comme chez Rimbaud dans son exploration de l'inconnu. Dans 10/11, les images de l'intérieur et des alentours sont le produit d'une poésie savante, alerte, intertextuelle et ingénieuse, d'une technique habile, celle d'un systématique humour. La tristesse est alors le fruit, non pas du trop-plein d'évocations dans les chambres et boudoirs chargés de souvenirs et de sensations et dans les paysages invitant à l'évasion et à l'imagination, mais plutôt d'une conscience aigüe de leur vide, que rien ne saurait combler. Après tout, même s'il ne se veut ni voyant ni guide dans la traversée des piliers de l'imagination, l'humoriste, nous confie Noguez, détient «la forme la plus achevée de la lucidité »(155).

\section{Ouvrages cités}

Amprimoz, Alexandre. 10/11. Sudbury : Prise de parole, 1979.

Barthes, Roland. S/Z. Oeuvres completes, tome 2. 1966-1973. Dir. Eric Marty. Paris : Seuil, 1994. 555-742. 
Bryson, Norman. «Introduction ». Bal, Mieke. Looking In : The Art of Viewing. Amsterdam : G+B Arts, 2001. 1-39.

Cotton, Charlotte. The Photograph as Contemporary Art, $2^{\mathrm{e}}$ edition. Londres : Thames and Hudson, 2009.

Eluard, Paul. «Liberté ». Poésie et vérité. Paris : Main à la plume, 1942. www.reseaucanope.fr/poetes-en-resistance/poetes/paul-eluard/liberte, consulté 15 février 2015.

Jameson, Fredric. Postmodernism, or the Cultural Logic of Late Capitalism. Durham : Duke UP, 1991.

Knight, Derek. «Feminism and Activism and their Influence on the 1970s and 1980s and Beyond». Cours 'Readings in Contemporary Art I: 1970-2000', Université Brock, automne 2012.

---. «Post-Modernism : The Shift from Aesthetic Autonomy to Cultural Irony and Paradox ». Cours 'Readings in Contemporary Art I: 1970-2000’, Université Brock, automne 2012.

---. « Trends in Canadian Art in the 1970s and Beyond ». Cours 'Readings in Contemporary Art I: 1970-2000', Université Brock, automne 2012.

Noguez, Dominique. «L'humour, ou la dernière des tristesses ». Etudes françaises 5.2 (1969) : $139-161$.

Morin, Christian. «Pour une définition sémiotique du discours humoristique ». Protée 30.3 (2002) : 91-98.

Rimbaud, Arthur. «Le bateau ivre ». Euvres complètes. Dir. Antoine Adam. Paris : Gallimard, 1972. 66-69.

---. «Les étrennes des orphelins ». Euvres complètes. Dir. Antoine Adam. Paris : Gallimard, 1972. 3-6.

Verlaine, Paul. «Clair de lune ». Euvres poétiques complètes. Dir. Y.-G. Le Dantec et Jacques Borel. Paris : NRF / La pléiade, 1962. 107.

Wood, Paul. «Inside the Whale: An Introduction to Contemporary Art». Themes in Contemporary Art. Dir. Gill Perry et Paul Wood. New Haven : Yale UP, 2004.

\section{Oeuvres d'art}

Dying Gaul, 2e s. AD, copie romaine, Capitoline Museum, Rome.

Friedrich, Casper David, La mer de glace, 1824, peinture à l'huile, 38 1/2 x 50 1/2 in, Kunsthalle, Hambourg. 
General Idea, Fin de siècle, 1994, épreuves Cibachromes, 79 x 55.7 cm., exposé au Centro Andaluz de Arte Contemporáneo, Séville.

---, 1984 Miss General Idea Pavilion: Snowbird, 1985, 27 bouteilles en plastique, exposé à Aurora Borealis, Montreal.

Goodwin, Betty, Red Sea, 1984, pastel à l'huile, pastel sec, huile, fusain sur papier vélum, Collection du Musée d'art contemporain, Montréal.

Koons, Jeff, Michael Jackson and Bubbles, 1988, céramique, 106.68 x 179.07 x 82.55 cm, Collection SFMOMA.

Manet, Edouard, Le bar aux Folies Bergère, 1882, peinture à l'huile, 96 x 130 cm., Institut Courtauld, Londres.

Mapplethorpe, Robert, Calla Lily, 1984, tirage platine, 25 1⁄2 x 22 in., The Robert Mapplethorpe Foundation.

Serrano, Andres, Untitled XIII (Ejaculate in Trajectory), 1989, Cibachrome, silicone, Plexiglas, 102 x 152 cm., Roush Fund for Contemporary Art.

Snow, Michael, Authorization, 1969, 5 tirages Polaroid n/b et adhésif à support toile sur miroir avec cadre en métal, 21.5 x 17.5 in., AMICA Library.

---, Plus Tard (détail), 1977, photographie sous plexiglass, 86.536 x $107.95 \mathrm{~cm}$. chacune, Galerie nationale du Canada, Ottawa.

---, Flight Stop, 1979, moule en fibre de verre et photographies, Eaton's Centre, Toronto.

Thomson, Tom, The Jack Pine, 1916-17, peinture à l'huile, 127.9 x 139.8, Galerie nationale du Canada, Ottawa.

Van Gogh, Vincent, Les vieux souliers aux lacets, 1885-87, peinture à l'huile, 14.8 x 17.7 in., Vincent van Gogh Museum, Amsterdam.

Wall, Jeff, Picture for Women, 1979, épreuves Cibachromes transparents, 161,5 x 223,5 x 28,5 $\mathrm{cm}$; deux épreuves Cibachromes transparents jointes bord à bord et caisson lumineux. 204.5 x 142.5 cm., Centre Georges Pompidou, Paris.

---, The Pine on the Corner, 1990, transparent cibachrome, light box aluminium, 47 x 59 in., Vancouver Art Gallery.

Warhol, Andy, Diamond Dust Shoes (Diagonal), 1980-81, peinture polymère synthétique, poussière de diamant et encre pour sérigraphie sur toile, 90 x 70 in., The Andy Warhol Museum, Pittsburgh. 
Wieland, Joyce, Artist on Fire, 1983, peinture à l'huile, 107 x $130 \mathrm{~cm}$., Robert McLaughlin Gallery. 